\title{
Rate of convergence by Kantorovich-Szász type operators based on Brenke type polynomials
}

\author{
Tarul Garg ${ }^{1}$ Purshottam Narain Agrawal ${ }^{1}$ and Serkan Araci ${ }^{2 *}$
}

\author{
"Correspondence: \\ mtsrkn@hotmail.com \\ ${ }^{2}$ Department of Economics, Faculty \\ of Economics, Administrative and \\ Social Sciences, Hasan Kalyoncu \\ University, Gaziantep, 27410, Turkey \\ Full list of author information is \\ available at the end of the article
}

\begin{abstract}
The present paper deals with the approximation properties of the univariate operators which are the generalization of the Kantorovich-Szász type operators involving Brenke type polynomials. We investigate the order of convergence by using Peetre's K-functional and the Ditzian-Totik modulus of smoothness and study the degree of approximation of the univariate operators for continuous functions in a Lipschitz space, a Lipschitz type maximal function and a weighted space. The rate of approximation of functions having derivatives equivalent with a function of bounded variation is also obtained.
\end{abstract}

MSC: $41 \mathrm{~A} 10 ; 41 \mathrm{~A} 25 ; 41 \mathrm{~A} 36$

Keywords: Brenke type polynomials; Szász operator; Ditzian-Totik modulus of smoothness; derivative of bounded variation; Peetre's K-functional; rate of convergence

\section{Introduction}

Linear positive operators play an important role in the study of approximation theory. One of the best known among these operators is the Szász operator [1]

$$
S_{n}(f ; x)=e^{-n x} \sum_{k=0}^{\infty} \frac{(n x)^{k}}{k !} f\left(\frac{k}{n}\right), \quad x \geq 0, n \in \mathbb{N}
$$

a generalization of Bernstein polynomials to the infinite interval. Jakimovski and Leviatan [2] generalized Szász operators by means of the Appell polynomials. Subsequently, Ismail [3] gave another generalization of the Szász operators by involving the Sheffer polynomials. Varma et al. [4] presented yet another generalization of the Szász operators by utilizing the Brenke type polynomials and showed that these polynomials include the Appell polynomials and Gould-Hopper polynomials as special cases. Varma and Taşdelen [5] determined a link between the Szász operators and the discrete orthogonal polynomials, e.g., Charlier polynomials, and established the Korovkin type theorem and the rate of convergence by means of the classical modulus of continuity. Taşdelen et al. [6] introduced a Kantorovich variant of the Szász operators based on Brenke type polynomials and discussed the order of convergence with the aid of the moduli of continuity and Peetre's K-

(c) The Author(s) 2017. This article is distributed under the terms of the Creative Commons Attribution 4.0 International License (http://creativecommons.org/licenses/by/4.0/), which permits unrestricted use, distribution, and reproduction in any medium, provided you give appropriate credit to the original author(s) and the source, provide a link to the Creative Commons license, and indicate if changes were made. 
functional. Öksüzer et al. [7] estimated the rate of convergence for functions of bounded variation for these operators by means of some results of probability theory. Later on, Aktaş et al. [8] considered a Stancu type modification of the Szász Kantorovich operators involving Brenke type polynomials and obtained the degree of approximation by means of the classical modulus of continuity and Peetre's K-functional. A Voronovskaya type theorem for the considered operators including Gould-Hopper polynomials was also proven. Mursaleen and Ansari [9] presented a Chlodowsky type generalization of Szász operators defined by using Brenke type polynomials and studied the order of convergence for functions in a weighted space besides other classical approximation results.

Recently, for $f \in C_{E}[0, \infty):=\left\{f \in C[0, \infty):|f(t)| \leq a e^{b t}, a \in \mathbb{R}^{+}, b \in \mathbb{R}\right\}$, Atakut and Büyükyazici [10] considered the Kantorovich-Szász type operators involving Brenke type polynomials as

$$
L_{n}^{\alpha_{n}, \beta_{n}}(f ; x)=\frac{\beta_{n}}{A(1) B\left(\alpha_{n} x\right)} \sum_{k=0}^{\infty} p_{k}\left(\alpha_{n} x\right) \int_{k / \beta_{n}}^{(k+1) / \beta_{n}} f(t) d t,
$$

where $\left\{\alpha_{n}\right\},\left\{\beta_{n}\right\}$ are strictly increasing sequences of positive numbers such that

$$
\lim _{n \rightarrow \infty} \frac{1}{\beta_{n}}=0, \quad \frac{\alpha_{n}}{\beta_{n}}=1+O\left(\frac{1}{\beta_{n}}\right), \quad \text { as } n \rightarrow \infty
$$

and $p_{k}(x)=\sum_{r=0}^{\infty} a_{k-r} b_{r} x^{r}, k=0,1,2, \ldots$, are Brenke type polynomials having the following generating function:

$$
A(t) B(x t)=\sum_{k=0}^{\infty} p_{k}(x) t^{k}
$$

where $A(t), B(t)$ are analytic functions such that

$$
\begin{aligned}
& A(t)=\sum_{r=0}^{\infty} a_{r} t^{r}, \quad a_{0} \neq 0, \\
& B(t)=\sum_{r=0}^{\infty} b_{r} t^{r}, \quad b_{r} \neq 0(r \geq 0)
\end{aligned}
$$

hold. Assume that

(i) $A(1) \neq 0, \frac{a_{k-r} b_{r}}{A(1)} \geq 0$ for $0 \leq r \leq k, k=0,1,2, \ldots$,

(ii) $B:[0, \infty) \rightarrow(0, \infty)$,

(iii) (3) and the power series (4) converges for $|t|<R(R>1)$,

(iv) $\lim _{y \rightarrow \infty} \frac{B^{(k)}(y)}{B(y)}=1$ for $k=1,2,3,4$.

Atakut and Büyükyazici [10] studied the order of convergence of the operators defined in (1). For the special case $\alpha_{n}=\beta_{n}=n$, the operators (1) are reduced to the Kantorovich variant of a generalization of Szász operators based on the Brenke type polynomials. For some other significant papers dealing with the generalization of Szász type operators, we refer to [11-13].

In the present paper we establish the order of approximation of the operators (1) by using Peetre's K-functional and the Ditzian-Totik modulus of smoothness. Also, we study 
the rate of convergence of these operators for a Lipschitz space, a Lipschitz type maximal function and a weighted space. The rate of approximation of functions having derivatives equivalent to a function of bounded variation is also obtained.

\section{Preliminaries}

To examine the approximation properties of the operator $L_{n}^{\alpha_{n}, \beta_{n}}$ defined in (1), we give some basic results in the form of lemmas as follows.

Lemma 2.1 ([10]) For $x \in[0, \infty)$, we have

(i) $L_{n}^{\alpha_{n}, \beta_{n}}(1 ; x)=1$,

(ii) $L_{n}^{\alpha_{n}, \beta_{n}}(t ; x)=\frac{\alpha_{n}}{\beta_{n}} \frac{B^{\prime}\left(\alpha_{n} x\right)}{B\left(\alpha_{n} x\right)} x+\frac{1}{\beta_{n}} \frac{2 A^{\prime}(1)+A(1)}{2 A(1)}$,

(iii) $\quad L_{n}^{\alpha_{n}, \beta_{n}}\left(t^{2} ; x\right)=\frac{\alpha_{n}^{2}}{\beta_{n}^{2}} \frac{B^{\prime \prime}\left(\alpha_{n} x\right)}{B\left(\alpha_{n} x\right)} x^{2}+\frac{2 \alpha_{n}}{\beta_{n}^{2}} \frac{A^{\prime}(1)+A(1)}{A(1)} \frac{B^{\prime}\left(\alpha_{n} x\right)}{B\left(\alpha_{n} x\right)} x$

$$
+\frac{1}{3 \beta_{n}^{2}} \frac{3 A^{\prime \prime}(1)+6 A^{\prime}(1)+A(1)}{A(1)}
$$

(iv) $L_{n}^{\alpha_{n}, \beta_{n}}\left(t^{3} ; x\right)=\frac{\alpha_{n}^{3}}{\beta_{n}^{3}} \frac{B^{\prime \prime \prime}\left(\alpha_{n} x\right)}{B\left(\alpha_{n} x\right)} x^{3}+\frac{3 \alpha_{n}^{2}}{2 \beta_{n}^{3}} \frac{2 A^{\prime}(1)+3 A(1)}{A(1)} \frac{B^{\prime \prime}\left(\alpha_{n} x\right)}{B\left(\alpha_{n} x\right)} x^{2}$

$$
\begin{aligned}
& +\frac{\alpha_{n}}{2 \beta_{n}^{3}} \frac{6 A^{\prime \prime}(1)+18 A^{\prime}(1)+7 A(1)}{A(1)} \frac{B^{\prime}\left(\alpha_{n} x\right)}{B\left(\alpha_{n} x\right)} x \\
& +\frac{1}{4 \beta_{n}^{3}} \frac{4 A^{\prime \prime \prime}(1)+18 A^{\prime \prime}(1)+14 A^{\prime}(1)+A(1)}{A(1)},
\end{aligned}
$$

(v) $L_{n}^{\alpha_{n}, \beta_{n}}\left(t^{4} ; x\right)=\frac{\alpha_{n}^{4}}{\beta_{n}^{4}} \frac{B^{(4)}\left(\alpha_{n} x\right)}{B\left(\alpha_{n} x\right)} x^{4}+\frac{4 \alpha_{n}^{3}}{\beta_{n}^{4}} \frac{A^{\prime}(1)+2 A(1)}{A(1)} \frac{B^{\prime \prime \prime}\left(\alpha_{n} x\right)}{B\left(\alpha_{n} x\right)} x^{3}$

$$
\begin{aligned}
& +\frac{3 \alpha_{n}^{2}}{\beta_{n}^{4}} \frac{2 A^{\prime \prime}(1)+8 A^{\prime}(1)+5 A(1)}{A(1)} \frac{B^{\prime \prime}\left(\alpha_{n} x\right)}{B\left(\alpha_{n} x\right)} x^{2} \\
& +\frac{\alpha_{n}}{\beta_{n}^{4}} \frac{4 A^{\prime \prime \prime}(1)+24 A^{\prime \prime}(1)+30 A^{\prime}(1)+6 A(1)}{A(1)} \frac{B^{\prime}\left(\alpha_{n} x\right)}{B\left(\alpha_{n} x\right)} x \\
& +\frac{1}{5 \beta_{n}^{4}} \frac{5 A^{(4)}(1)+40 A^{\prime \prime \prime}(1)+75 A^{\prime \prime}(1)+30 A^{\prime}(1)+A(1)}{A(1)} .
\end{aligned}
$$

As a consequence of Lemma 2.1, we have the following.

Lemma 2.2 For $x \in[0, \infty)$,

(i) $L_{n}^{\alpha_{n}, \beta_{n}}(t-x ; x)=\left(\frac{\alpha_{n}}{\beta_{n}} \frac{B^{\prime}\left(\alpha_{n} x\right)}{B\left(\alpha_{n} x\right)}-1\right) x+\frac{1}{\beta_{n}} \frac{2 A^{\prime}(1)+A(1)}{2 A(1)}$,

(ii) $L_{n}^{\alpha_{n}, \beta_{n}}\left((t-x)^{2} ; x\right)=\left(\frac{\alpha_{n}^{2}}{\beta_{n}^{2}} \frac{B^{\prime \prime}\left(\alpha_{n} x\right)}{B\left(\alpha_{n} x\right)}-\frac{2 \alpha_{n}}{\beta_{n}} \frac{B^{\prime}\left(\alpha_{n} x\right)}{B\left(\alpha_{n} x\right)}+1\right) x^{2}$

$$
\begin{aligned}
& +\left(\frac{2 \alpha_{n}}{\beta_{n}^{2}} \frac{A^{\prime}(1)+A(1)}{A(1)} \frac{B^{\prime}\left(\alpha_{n} x\right)}{B\left(\alpha_{n} x\right)}-\frac{1}{\beta_{n}} \frac{2 A^{\prime}(1)+A(1)}{2 A(1)}\right) x \\
& +\frac{1}{3 \beta_{n}^{2}} \frac{3 A^{\prime \prime}(1)+6 A^{\prime}(1)+A(1)}{A(1)},
\end{aligned}
$$




$$
\text { (iii) } \begin{aligned}
L_{n}^{\alpha_{n}, \beta_{n}}\left((t-x)^{4} ; x\right)= & \left(\frac{\alpha_{n}^{4}}{\beta_{n}^{4}} \frac{B^{(4)}\left(\alpha_{n} x\right)}{B\left(\alpha_{n} x\right)}-\frac{4 \alpha_{n}^{3}}{\beta_{n}^{3}} \frac{B^{\prime \prime \prime}\left(\alpha_{n} x\right)}{B\left(\alpha_{n} x\right)}+\frac{6 \alpha_{n}^{2}}{\beta_{n}^{2}} \frac{B^{\prime \prime}\left(\alpha_{n} x\right)}{B\left(\alpha_{n} x\right)}\right. \\
& \left.-\frac{4 \alpha_{n}}{\beta_{n}} \frac{B^{\prime}\left(\alpha_{n} x\right)}{B\left(\alpha_{n} x\right)}+1\right) x^{4}+\left(\frac{4 \alpha_{n}^{3}}{\beta_{n}^{4}} \frac{A^{\prime}(1)+2 A(1)}{A(1)} \frac{B^{\prime \prime \prime}\left(\alpha_{n} x\right)}{B\left(\alpha_{n} x\right)}\right. \\
& -\frac{6 \alpha_{n}^{2}}{\beta_{n}^{3}} \frac{2 A^{\prime}(1)+3 A(1)}{A(1)} \frac{B^{\prime \prime}\left(\alpha_{n} x\right)}{B\left(\alpha_{n} x\right)}+\frac{12 \alpha_{n}}{\beta_{n}^{2}} \frac{A^{\prime}(1)+A(1)}{A(1)} \frac{B^{\prime}\left(\alpha_{n} x\right)}{B\left(\alpha_{n} x\right)} \\
& \left.-\frac{2}{\beta_{n}} \frac{2 A^{\prime}(1)+A(1)}{A(1)}\right) x^{3}+\left(\frac{3 \alpha_{n}^{2}}{\beta_{n}^{4}} \frac{2 A^{\prime \prime}(1)+8 A^{\prime}(1)+5 A(1)}{A(1)}\right. \\
& \times \frac{B^{\prime \prime}\left(\alpha_{n} x\right)}{B\left(\alpha_{n} x\right)}-\frac{2 \alpha_{n}}{\beta_{n}^{3}} \frac{6 A^{\prime \prime}(1)+18 A^{\prime}(1)+7 A(1)}{A(1)} \frac{B^{\prime}\left(\alpha_{n} x\right)}{B\left(\alpha_{n} x\right)} \\
& \left.+\frac{2}{\beta_{n}^{2}} \frac{3 A^{\prime \prime}(1)+6 A^{\prime}(1)+A(1)}{A(1)}\right) x^{2} \\
& +\left(\frac{\alpha_{n}}{\beta_{n}^{4}} \frac{4 A^{\prime \prime \prime}(1)+24 A^{\prime \prime}(1)+30 A^{\prime}(1)+6 A(1)}{A(1)} \frac{B^{\prime}\left(\alpha_{n} x\right)}{B\left(\alpha_{n} x\right)}\right. \\
& \left.-\frac{1}{\beta_{n}^{3}} \frac{4 A^{\prime \prime \prime}(1)+18 A^{\prime \prime}(1)+14 A^{\prime}(1)+A(1)}{A(1)}\right) x \\
& +\frac{1}{5 \beta_{n}^{4}} \frac{5 A^{(4)}(1)+40 A^{\prime \prime \prime}(1)+75 A^{\prime \prime}(1)+30 A^{\prime}(1)+A(1)}{A(1)}
\end{aligned}
$$

\section{Local theorem of approximation}

In what follows, let $L_{n}^{\alpha_{n}, \beta_{n}}(t-x ; x)=\gamma_{\alpha_{n}, \beta_{n}}(x)$ and $L_{n}^{\alpha_{n}, \beta_{n}}\left((t-x)^{2} ; x\right)=\delta_{\alpha_{n}, \beta_{n}}^{2}(x)$. Further, $M$ denotes a constant not necessarily the same at each occurrence.

The Lipschitz type space [1] is defined as follows:

$$
\operatorname{Lip}_{M}^{*}(r):=\left\{f \in C[0, \infty):|f(t)-f(x)| \leq M_{f} \frac{|t-x|^{r}}{(t+x)^{r / 2}} ; x, t>0\right\}
$$

for some $M_{f}>0$ and $0<r \leq 1$. Several researchers have considered the approximation of functions in this space for different sequences of linear positive operators (cf. [12, 14-16] etc.).

Theorem 3.1 Let $f \in \operatorname{Lip}_{M}^{*}(r)$ and $r \in(0,1]$, then $\forall x>0$

$$
\left|L_{n}^{\alpha_{n}, \beta_{n}}(f ; x)-f(x)\right| \leq M_{f}\left(\frac{\delta_{\alpha_{n}, \beta_{n}}(x)}{\sqrt{x}}\right)^{r}
$$

Proof Since $f \in \operatorname{Lip}_{M}^{*}(r)$, by definition (5)

$$
|f(t)-f(x)| \leq M_{f} \frac{|t-x|^{r}}{(t+x)^{r / 2}}
$$

Operating by $L_{n}^{\alpha_{n}, \beta_{n}}$ on the above equation, we get

$$
\left|L_{n}^{\alpha_{n}, \beta_{n}}(f ; x)-f(x)\right| \leq M_{f} L_{n}^{\alpha_{n}, \beta_{n}}\left(\frac{|t-x|^{r}}{(t+x)^{r / 2}} ; x\right) .
$$


Now, applying Hölder's inequality with $p=2 / r, q=2 /(2-r)$ and using Lemma 2.1, we get

$$
\begin{aligned}
\left|L_{n}^{\alpha_{n}, \beta_{n}}(f ; x)-f(x)\right| & \leq M_{f}\left(L_{n}^{\alpha_{n}, \beta_{n}}\left(\frac{(t-x)^{2}}{(t+x)} ; x\right)\right)^{r / 2}\left(L_{n}^{\alpha_{n}, \beta_{n}}(1 ; x)\right)^{(2-r) / 2} \\
& \leq \frac{M_{f}}{x^{r / 2}}\left(L_{n}^{\alpha_{n}, \beta_{n}}\left((t-x)^{2} ; x\right)\right)^{r / 2}
\end{aligned}
$$

which gives the required result.

Let $\tilde{C}_{B}[0, \infty)$ denote the space of all real-valued bounded and uniformly continuous functions $f$ on $[0, \infty)$, endowed with the norm $\|f\|_{\infty}=\sup _{x \in[0, \infty)}|f(x)|$. Further, we obtain a local direct estimate for the operators (1) using the Lipschitz maximal function of order $r$ introduced by Lenze [17]

$$
\tilde{\omega}_{r}(f ; x)=\sup _{t \neq x, t \in[0, \infty)} \frac{|f(t)-f(x)|}{|t-x|^{r}},
$$

where $x \in[0, \infty)$ and $r \in(0,1]$. For contributions of researchers on approximation of functions in this space, we refer to $[12,14,15,18]$.

Theorem 3.2 Let $f \in \tilde{C}_{B}[0, \infty)$ and $r \in(0,1]$, then $\forall x \in[0, \infty)$

$$
\left|L_{n}^{\alpha_{n}, \beta_{n}}(f ; x)-f(x)\right| \leq \tilde{\omega}_{r}(f ; x)\left(\delta_{\alpha_{n}, \beta_{n}}(x)\right)^{r} .
$$

Proof By equation (6),

$$
|f(t)-f(x)| \leq \tilde{\omega}_{r}(f ; x)|t-x|^{r} .
$$

Applying the operator $L_{n}^{\alpha_{n}, \beta_{n}}$ on both sides of the above equation, and then using Lemma 2.1 and Hölder's inequality with $p=2 / r, q=2 /(2-r)$, we get

$$
\begin{aligned}
\left|L_{n}^{\alpha_{n}, \beta_{n}}(f ; x)-f(x)\right| & \leq \tilde{\omega}_{r}(f ; x) L_{n}^{\alpha_{n}, \beta_{n}}\left(|t-x|^{r} ; x\right) \\
& \leq \tilde{\omega}_{r}(f ; x)\left(L_{n}^{\alpha_{n}, \beta_{n}}\left((t-x)^{2} ; x\right)\right)^{r / 2}\left(L_{n}^{\alpha_{n}, \beta_{n}}(1 ; x)\right)^{(2-r) / 2} \\
& =\tilde{\omega}_{r}(f ; x)\left(\delta_{\alpha_{n}, \beta_{n}}(x)\right)^{r} .
\end{aligned}
$$

Thus, we get the desired result.

For $f \in \tilde{C}_{B}[0, \infty)$ and $\delta>0$, the first order modulus of continuity is defined as

$$
\omega(f ; \delta)=\sup _{0<|h| \leq \delta x, x+h \in[0, \infty)} \sup _{\mid}|f(x+h)-f(x)|,
$$

and Peetre's K-functional is given by

$$
K(f ; \delta)=\inf _{g \in \tilde{C}_{B}^{2}[0, \infty)}\left\{\|f-g\|_{\infty}+\delta\|g\|_{\tilde{C}_{B}^{2}}\right\},
$$


where $\tilde{C}_{B}^{2}[0, \infty)=\left\{g \in \tilde{C}_{B}[0, \infty): g^{\prime}, g^{\prime \prime} \in \tilde{C}_{B}[0, \infty)\right\}$ with norm $\|g\|_{\tilde{C}_{B}^{2}}=\|g\|_{\infty}+\left\|g^{\prime}\right\|_{\infty}+$ $\left\|g^{\prime \prime}\right\|_{\infty}$. Also

$$
K(f ; \delta) \leq M\left(\omega_{2}(f ; \sqrt{\delta})+\min \{1, \delta\}\|f\|_{\infty}\right)
$$

holds for all $\delta>0$ and where $\omega_{2}$ is the second order modulus of smoothness of $f \in$ $\tilde{C}_{B}[0, \infty)$, which is defined as follows:

$$
\omega_{2}(f ; \delta)=\sup _{0<|h| \leq \delta} \sup _{x, x+2 h \in[0, \infty)}|f(x+2 h)-2 f(x+h)+f(x)| .
$$

Theorem 3.3 Iff $\in \tilde{C}_{B}[0, \infty)$, then

$$
\left|L_{n}^{\alpha_{n}, \beta_{n}}(f ; x)-f(x)\right| \leq 4 K\left(f ; k_{\alpha_{n}, \beta_{n}}(x)\right)+\omega\left(f, \gamma_{\alpha_{n}, \beta_{n}}(x)\right)
$$

where $k_{\alpha_{n}, \beta_{n}}(x)=\left(\delta_{\alpha_{n}, \beta_{n}}^{2}(x)+\gamma_{\alpha_{n}, \beta_{n}}^{2}(x)\right) / 4$. Furthermore,

$$
\begin{aligned}
\left|L_{n}^{\alpha_{n}, \beta_{n}}(f ; x)-f(x)\right| \leq & M\left(\omega_{2}\left(f ; \sqrt{k_{\alpha_{n}, \beta_{n}}(x)}\right)+\min \left\{1, k_{\alpha_{n}, \beta_{n}}(x)\right\}\|f\|_{\infty}\right) \\
& +\omega\left(f,\left|\gamma_{\alpha_{n}, \beta_{n}}(x)\right|\right) .
\end{aligned}
$$

Proof For $f \in \tilde{C}_{B}[0, \infty)$, we define the auxiliary operator as follows:

$$
\tilde{L}_{n}^{\alpha_{n}, \beta_{n}}(f ; x)=L_{n}^{\alpha_{n}, \beta_{n}}(f ; x)-f\left(\frac{\alpha_{n}}{\beta_{n}} \frac{B^{\prime}\left(\alpha_{n} x\right)}{B\left(\alpha_{n} x\right)} x+\frac{1}{\beta_{n}} \frac{2 A^{\prime}(1)+A(1)}{2 A(1)}\right)+f(x) .
$$

After taking the modulus of both sides and applying Lemma 2.1, we obtain

$$
\begin{aligned}
\left|\tilde{L}_{n}^{\alpha_{n}, \beta_{n}}(f ; x)\right| \leq & \left|L_{n}^{\alpha_{n}, \beta_{n}}(f ; x)\right|+\left|f\left(\frac{\alpha_{n}}{\beta_{n}} \frac{B^{\prime}\left(\alpha_{n} x\right)}{B\left(\alpha_{n} x\right)} x+\frac{1}{\beta_{n}} \frac{2 A^{\prime}(1)+A(1)}{2 A(1)}\right)\right| \\
& +|f(x)| \\
\leq & \|f\|_{\infty}\left|L_{n}^{\alpha_{n}, \beta_{n}}(1 ; x)\right|+\|f\|_{\infty}+\|f\|_{\infty} \\
= & 3\|f\|_{\infty} .
\end{aligned}
$$

Now, by Lemma 2.1, we get

$$
\tilde{L}_{n}^{\alpha_{n}, \beta_{n}}(t ; x)=x
$$

and therefore

$$
\tilde{L}_{n}^{\alpha_{n}, \beta_{n}}(t-x ; x)=0
$$

Let $g \in \tilde{C}_{B}^{2}[0, \infty)$, then using Taylor's theorem we can write

$$
g(t)=g(x)+(t-x) g^{\prime}(x)+\int_{x}^{t}(t-u) g^{\prime \prime}(u) d u
$$


Operating $\tilde{L}_{n}^{\alpha_{n}, \beta_{n}}$ on the above equation and using (10), we get

$$
\begin{aligned}
\tilde{L}_{n}^{\alpha_{n}, \beta_{n}}(g ; x)-g(x) \\
=\tilde{L}_{n}^{\alpha_{n}, \beta_{n}}\left(\int_{x}^{t}(t-u) g^{\prime \prime}(u) d u ; x\right) \\
=L_{n}^{\alpha_{n}, \beta_{n}}\left(\int_{x}^{t}(t-u) g^{\prime \prime}(u) d u ; x\right) \\
\quad-\int_{x}^{\frac{\alpha_{n}}{\beta_{n}} \frac{B^{\prime}\left(\alpha_{n} x\right)}{B\left(\alpha_{n} x\right)} x+\frac{1}{\beta_{n}} \frac{2 A^{\prime}(1)+A(1)}{2 A(1)}}\left(\frac{\alpha_{n}}{\beta_{n}} \frac{B^{\prime}\left(\alpha_{n} x\right)}{B\left(\alpha_{n} x\right)} x+\frac{1}{\beta_{n}} \frac{2 A^{\prime}(1)+A(1)}{2 A(1)}-u\right) g^{\prime \prime}(u) d u .
\end{aligned}
$$

Now taking the modulus on both sides, we obtain

$$
\begin{aligned}
& \left|\tilde{L}_{n}^{\alpha_{n}, \beta_{n}}(g ; x)-g(x)\right| \\
& \leq\left|L_{n}^{\alpha_{n}, \beta_{n}}\left(\int_{x}^{t}(t-u) g^{\prime \prime}(u) d u ; x\right)\right| \\
& +\left|\int_{x}^{\frac{\alpha_{n}}{\beta_{n}} \frac{B^{\prime}\left(\alpha_{n} x\right)}{B\left(\alpha_{n} x\right)} x+\frac{1}{\beta_{n}} \frac{2 A^{\prime}(1)+A(1)}{2 A(1)}}\left(\frac{\alpha_{n}}{\beta_{n}} \frac{B^{\prime}\left(\alpha_{n} x\right)}{B\left(\alpha_{n} x\right)} x+\frac{1}{\beta_{n}} \frac{2 A^{\prime}(1)+A(1)}{2 A(1)}-u\right) g^{\prime \prime}(u) d u\right| \\
& \leq L_{n}^{\alpha_{n}, \beta_{n}}\left(\left|\int_{x}^{t}\right|(t-u)|| g^{\prime \prime}(u)|d u| ; x\right) \\
& +\left|\left(\int_{x}^{\frac{\alpha_{n}}{\beta_{n}} \frac{B^{\prime}\left(\alpha_{n} x\right)}{B\left(\alpha_{n} x\right)} x+\frac{1}{\beta_{n}} \frac{2 A^{\prime}(1)+A(1)}{2 A(1)}}\left|\frac{\alpha_{n}}{\beta_{n}} \frac{B^{\prime}\left(\alpha_{n} x\right)}{B\left(\alpha_{n} x\right)} x+\frac{1}{\beta_{n}} \frac{2 A^{\prime}(1)+A(1)}{2 A(1)}-u\right|\left|g^{\prime \prime}(u)\right| d u\right)\right| \\
& \leq\left\|g^{\prime \prime}\right\|_{\infty}\left\{L_{n}^{\alpha_{n}, \beta_{n}}\left(\left|\int_{x}^{t}\right|(t-u)|d u| ; x\right)\right. \\
& \left.+\left|\left(\int_{x}^{\frac{\alpha_{n}}{\beta_{n}} \frac{B^{\prime}\left(\alpha_{n} x\right)}{B\left(\alpha_{n} x\right)} x+\frac{1}{\beta_{n}} \frac{2 A^{\prime}(1)+A(1)}{2 A(1)}}\left|\frac{\alpha_{n}}{\beta_{n}} \frac{B^{\prime}\left(\alpha_{n} x\right)}{B\left(\alpha_{n} x\right)} x+\frac{1}{\beta_{n}} \frac{2 A^{\prime}(1)+A(1)}{2 A(1)}-u\right| d u\right)\right|\right\} .
\end{aligned}
$$

Therefore, by using $\|g\|_{\tilde{C}_{B}^{2}}=\|g\|_{\infty}+\left\|g^{\prime}\right\|_{\infty}+\left\|g^{\prime \prime}\right\|_{\infty}$,

$$
\begin{aligned}
& \left|\tilde{L}_{n}^{\alpha_{n}, \beta_{n}}(g ; x)-g(x)\right| \\
& \quad \leq\|g\|_{\tilde{C}_{B}^{2}}\left\{L_{n}^{\alpha_{n}, \beta_{n}}\left((t-x)^{2} ; x\right)+\left(\frac{\alpha_{n}}{\beta_{n}} \frac{B^{\prime}\left(\alpha_{n} x\right)}{B\left(\alpha_{n} x\right)} x+\frac{1}{\beta_{n}} \frac{2 A^{\prime}(1)+A(1)}{2 A(1)}-x\right)^{2}\right\} \\
& \quad \leq\|g\|_{\tilde{C}_{B}^{2}}\left\{L_{n}^{\alpha_{n}, \beta_{n}}\left((t-x)^{2} ; x\right)+\left(L_{n}^{\alpha_{n}, \beta_{n}}(t-x ; x)\right)^{2}\right\} \\
& \quad=\|g\|_{\tilde{C}_{B}^{2}}^{2}\left\{\delta_{\alpha_{n}, \beta_{n}}^{2}(x)+\gamma_{\alpha_{n}, \beta_{n}}^{2}(x)\right\} .
\end{aligned}
$$

Now, using the definition of auxiliary operators (8), we have

$$
\begin{aligned}
& \left|L_{n}^{\alpha_{n}, \beta_{n}}(f ; x)-f(x)\right| \\
& \leq\left|\tilde{L}_{n}^{\alpha_{n}, \beta_{n}}(f ; x)-f(x)+f\left(\frac{\alpha_{n}}{\beta_{n}} \frac{B^{\prime}\left(\alpha_{n} x\right)}{B\left(\alpha_{n} x\right)} x+\frac{1}{\beta_{n}} \frac{2 A^{\prime}(1)+A(1)}{2 A(1)}\right)-f(x)\right| \\
& \leq\left|\tilde{L}_{n}^{\alpha_{n}, \beta_{n}}(f-g ; x)\right|+\left|\tilde{L}_{n}^{\alpha_{n}, \beta_{n}}(g ; x)-g(x)\right|+|g(x)-f(x)| \\
& \quad+\left|f\left(\frac{\alpha_{n}}{\beta_{n}} \frac{B^{\prime}\left(\alpha_{n} x\right)}{B\left(\alpha_{n} x\right)} x+\frac{1}{\beta_{n}} \frac{2 A^{\prime}(1)+A(1)}{2 A(1)}\right)-f(x)\right| .
\end{aligned}
$$


Combining (7), (9) and (11) with the above equation, we get

$$
\begin{aligned}
& \left|L_{n}^{\alpha_{n}, \beta_{n}}(f ; x)-f(x)\right| \\
& \quad \leq 4\|f-g\|_{\infty}+\|g\|_{\tilde{C}_{B}^{2}}\left\{\delta_{\alpha_{n}, \beta_{n}}^{2}(x)+\gamma_{\alpha_{n}, \beta_{n}}^{2}(x)\right\}+\omega\left(f,\left|\gamma_{\alpha_{n}, \beta_{n}}(x)\right|\right) \\
& \quad \leq 4\|f-g\|_{\infty}+\|g\|_{\tilde{C}_{B}^{2}} 4 k_{\alpha_{n}, \beta_{n}}(x)+\omega\left(f,\left|\gamma_{\alpha_{n}, \beta_{n}}(x)\right|\right),
\end{aligned}
$$

and, after taking the infimum on the right-hand side over all $g \in \tilde{C}_{B}^{2}$, we obtain

$$
\begin{aligned}
\left|L_{n}^{\alpha_{n}, \beta_{n}}(f ; x)-f(x)\right| \\
\leq 4 K\left(f ; k_{\alpha_{n}, \beta_{n}}(x)\right)+\omega\left(f,\left|\gamma_{\alpha_{n}, \beta_{n}}(x)\right|\right) \\
\leq M\left(\omega_{2}\left(f ; \sqrt{k_{\alpha_{n}, \beta_{n}}(x)}\right)+\min \left\{1, k_{\alpha_{n}, \beta_{n}}(x)\right\}\|f\|_{\infty}\right) \\
\quad+\omega\left(f,\left|\gamma_{\alpha_{n}, \beta_{n}}(x)\right|\right) .
\end{aligned}
$$

This completes the proof.

Theorem 3.4 Let $f \in \tilde{C}_{B}^{1}[0, \infty)$, then $\forall x \geq 0$ and $\delta>0$,

$$
\left|L_{n}^{\alpha_{n}, \beta_{n}}(f ; x)-f(x)\right| \leq\left\{\left|f^{\prime}(x)\right|+2 \omega\left(f^{\prime}, \frac{\delta_{\alpha_{n}, \beta_{n}}(x)}{2}\right)\right\} \delta_{\alpha_{n}, \beta_{n}}(x) .
$$

Proof Since $f \in \tilde{C}_{B}^{1}[0, \infty)$, we may write

$$
f(t)-f(x)=f^{\prime}(x)(t-x)+\int_{x}^{t}\left(f^{\prime}(u)-f^{\prime}(x)\right) d u
$$

Now, using the well-known property of modulus of continuity, for $\delta>0$ and $f \in \tilde{C}_{B}^{1}[0, \infty)$, we get

$$
\left|\left(f^{\prime}(u)-f^{\prime}(x)\right)\right| \leq \omega\left(f^{\prime}, \delta\right)\left(\frac{|u-x|}{\delta}+1\right)
$$

hence

$$
\left|\int_{x}^{t}\left(f^{\prime}(u)-f^{\prime}(x)\right) d u\right| \leq \omega\left(f^{\prime}, \delta\right)\left(\frac{(t-x)^{2}}{2 \delta}+|t-x|\right) .
$$

Therefore,

$$
\begin{aligned}
\left|L_{n}^{\alpha_{n}, \beta_{n}}(f ; x)-f(x)\right| \leq & \left|f^{\prime}(x)\right| L_{n}^{\alpha_{n}, \beta_{n}}(|t-x| ; x) \\
& +\omega\left(f^{\prime}, \delta\right)\left(\frac{1}{2 \delta} L_{n}^{\alpha_{n}, \beta_{n}}\left((t-x)^{2} ; x\right)+L_{n}^{\alpha_{n}, \beta_{n}}(|t-x| ; x)\right) .
\end{aligned}
$$


After applying the Cauchy-Schwarz inequality, we have

$$
\begin{aligned}
\left|L_{n}^{\alpha_{n}, \beta_{n}}(f ; x)-f(x)\right| \leq & \left(\left|f^{\prime}(x)\right|+\omega\left(f^{\prime}, \delta\right)\right) \sqrt{L_{n}^{\alpha_{n}, \beta_{n}}\left((t-x)^{2} ; x\right)} \sqrt{L_{n}^{\alpha_{n}, \beta_{n}}(1 ; x)} \\
& +\omega\left(f^{\prime}, \delta\right)\left(\frac{1}{2 \delta} L_{n}^{\alpha_{n}, \beta_{n}}\left((t-x)^{2} ; x\right)\right) \\
= & \left(\left|f^{\prime}(x)\right|+\omega\left(f^{\prime}, \delta\right)\right) \delta_{\alpha_{n}, \beta_{n}}(x)+\omega\left(f^{\prime}, \delta\right)\left(\frac{\delta_{\alpha_{n}, \beta_{n}}^{2}(x)}{2 \delta}\right) .
\end{aligned}
$$

Choosing $\delta=\frac{1}{2} \delta_{\alpha_{n}, \beta_{n}}(x)$, we get our result.

Let $\phi(x)=\sqrt{x}$. For $f \in \tilde{C}_{B}[0, \infty)$, the Ditzian-Totik modulus of smoothness of first order is given by

$$
\omega_{\phi}(f ; \delta)=\sup _{0<h \leq \delta}\left\{\left|f\left(x+\frac{h \phi(x)}{2}\right)-f\left(x-\frac{h \phi(x)}{2}\right)\right| ; x \pm \frac{h \phi(x)}{2} \in[0, \infty)\right\},
$$

and appropriate Peetre's K-functional is defined by

$$
K_{\phi}(f ; \delta)=\inf _{g \in W_{\phi}[0, \infty)}\left\{\|f-g\|_{\infty}+\delta\left\|\phi g^{\prime}\right\|_{\infty}\right\}, \quad \delta>0
$$

where $W_{\phi}[0, \infty):=\left\{g: g \in A C_{\text {loc }}[0, \infty),\left\|\phi g^{\prime}\right\|_{\infty}<\infty\right\}$ and $g \in A C_{\text {loc }}[0, \infty)$ means that $g$ is differentiable and absolutely continuous on every compact subset $[a, b]$ of $[0, \infty)$. It is known from [19] that there exists a constant $M>0$ such that

$$
M^{-1} \omega_{\phi}(f ; \delta) \leq K_{\phi}(f ; \delta) \leq M \omega_{\phi}(f ; \delta) .
$$

Now, we find the order of approximation of the operator (1) by means of the Ditzian-Totik modulus of smoothness.

Theorem 3.5 For any $f \in \tilde{C}_{B}[0, \infty)$ and $x \in(0, \infty)$,

$$
\left|L_{n}^{\alpha_{n}, \beta_{n}}(f ; x)-f(x)\right| \leq M \omega_{\phi}\left(f ; \frac{\delta_{\alpha_{n}, \beta_{n}}(x)}{\sqrt{x}}\right)
$$

Proof By Taylor's theorem, for any $g \in W_{\phi}[0, \infty)$, we get

$$
\begin{aligned}
g(t) & =g(x)+\int_{x}^{t} g^{\prime}(u) d u \\
& =g(x)+\int_{x}^{t} \frac{g^{\prime}(u) \phi(u)}{\phi(u)} d u,
\end{aligned}
$$

therefore,

$$
\begin{aligned}
|g(t)-g(x)| & \leq\left\|\phi g^{\prime}\right\|_{\infty}\left|\int_{x}^{t} \frac{1}{\phi(u)} d u\right| \\
& =\left\|\phi g^{\prime}\right\|_{\infty} \cdot 2|\sqrt{t}-\sqrt{x}| \\
& =2\left\|\phi g^{\prime}\right\|_{\infty} \frac{|t-x|}{\sqrt{t}+\sqrt{x}}
\end{aligned}
$$


which gives

$$
\begin{aligned}
|g(t)-g(x)| & \leq 2\left\|\phi g^{\prime}\right\|_{\infty} \frac{|t-x|}{\sqrt{x}} \\
& =2\left\|\phi g^{\prime}\right\|_{\infty} \frac{|t-x|}{\phi(x)} .
\end{aligned}
$$

For any $g \in W_{\phi}[0, \infty)$, using Lemma 2.1 and the above equation, we get

$$
\begin{aligned}
\left|L_{n}^{\alpha_{n}, \beta_{n}}(f ; x)-f(x)\right| & \leq\left|L_{n}^{\alpha_{n}, \beta_{n}}(f-g ; x)\right|+\left|L_{n}^{\alpha_{n}, \beta_{n}}(g ; x)-g(x)\right|+|g(x)-f(x)| \\
& \leq 2\|f-g\|_{\infty}+\frac{2\left\|\phi g^{\prime}\right\|_{\infty}}{\phi(x)} L_{n}^{\alpha_{n}, \beta_{n}}(|t-x| ; x) .
\end{aligned}
$$

After applying the Cauchy-Schwarz inequality, we obtain

$$
\begin{aligned}
\left|L_{n}^{\alpha_{n}, \beta_{n}}(f ; x)-f(x)\right| & \leq 2\|f-g\|_{\infty}+\frac{2\left\|\phi g^{\prime}\right\|_{\infty}}{\phi(x)}\left[L_{n}^{\alpha_{n}, \beta_{n}}\left((t-x)^{2} ; x\right)\right]^{1 / 2} \\
& =2\|f-g\|_{\infty}+\frac{2\left\|\phi g^{\prime}\right\|_{\infty}}{\phi(x)} \delta_{\alpha_{n}, \beta_{n}}(x) .
\end{aligned}
$$

Taking the infimum on the right-hand side over all $g \in W_{\phi}[0, \infty)$, we get

$$
\left|L_{n}^{\alpha_{n}, \beta_{n}}(f ; x)-f(x)\right| \leq 2 K_{\phi}\left(f ; \frac{\delta_{\alpha_{n}, \beta_{n}}(x)}{\sqrt{x}}\right)
$$

which leads us to the required result with the help of the relation between Peetre's Kfunctional and the Ditzian-Totik modulus of smoothness as given in (12).

\section{Weighted approximation}

For $\gamma>0$, let $C_{\gamma}[0, \infty):=\left\{f \in C[0, \infty):|f(t)| \leq M_{f}\left(1+t^{\gamma}\right), \forall t \geq 0\right\}$ equipped with the norm

$$
\|f\|_{\gamma}=\sup _{t \in[0, \infty)} \frac{|f(t)|}{\left(1+t^{\gamma}\right)} .
$$

Further, let $C_{2}^{*}[0, \infty)$ be the subspace of $C_{2}[0, \infty)$ consisting of functions $f$ such that $\lim _{t \rightarrow \infty} \frac{f(t)}{\left(1+t^{2}\right)}$ exists. Such type of spaces is studied by many mathematicians as described in $[20-22]$ etc.

Theorem 4.1 For each $f \in C_{2}^{*}[0, \infty)$ and $r>0$,

$$
\lim _{n \rightarrow \infty} \sup _{x \in[0, \infty)} \frac{\left|L_{n}^{\alpha_{n}, \beta_{n}}(f ; x)-f(x)\right|}{\left(1+x^{2}\right)^{1+r}}=0
$$


Proof Let $x_{0}>0$ be arbitrary but fixed, then in view of (13) we get

$$
\begin{aligned}
& \sup _{x \in[0, \infty)} \frac{\left|L_{n}^{\alpha_{n}, \beta_{n}}(f ; x)-f(x)\right|}{\left(1+x^{2}\right)^{1+r}} \\
& \quad \leq \sup _{x \leq x_{0}} \frac{\left|L_{n}^{\alpha_{n}, \beta_{n}}(f ; x)-f(x)\right|}{\left(1+x^{2}\right)^{1+r}}+\sup _{x>x_{0}} \frac{\left|L_{n}^{\alpha_{n}, \beta_{n}}(f ; x)-f(x)\right|}{\left(1+x^{2}\right)^{1+r}} \\
& \quad \leq \sup _{x \leq x_{0}}\left\{\left|L_{n}^{\alpha_{n}, \beta_{n}}(f ; x)-f(x)\right|\right\}+\sup _{x>x_{0}} \frac{\left|L_{n}^{\alpha_{n}, \beta_{n}}(f ; x)\right|+|f(x)|}{\left(1+x^{2}\right)^{1+r}} .
\end{aligned}
$$

Since $|f(t)| \leq\|f\|_{2}\left(1+t^{2}\right)$, hence

$$
\begin{aligned}
\sup _{x \in[0, \infty)} \frac{\left|L_{n}^{\alpha_{n}, \beta_{n}}(f ; x)-f(x)\right|}{\left(1+x^{2}\right)^{1+r}} \leq & \left\|L_{n}^{\alpha_{n}, \beta_{n}}(f ; x)-f(x)\right\|_{C\left[0, x_{0}\right]} \\
& +\|f\|_{2} \sup _{x>x_{0}} \frac{\left|L_{n}^{\alpha_{n}, \beta_{n}}\left(1+t^{2} ; x\right)\right|}{\left(1+x^{2}\right)^{1+r}} \\
& +\sup _{x>x_{0}} \frac{|f(x)|}{\left(1+x^{2}\right)^{1+r}} \\
= & I_{1}+I_{2}+I_{3}, \quad \text { say. }
\end{aligned}
$$

By the Korovkin theorem, the sequence $\left\{L_{n}^{\alpha_{n}, \beta_{n}}(f ; x)\right\}$ converges uniformly to the function $f$ on every closed interval $[0, a]$, as $n \rightarrow \infty$, (cf. [10], p.1491), therefore for a given $\epsilon>0$, $\exists n_{1} \in \mathbb{N}$ such that

$$
I_{1}=\left\|L_{n}^{\alpha_{n}, \beta_{n}}(f ; x)-f(x)\right\|_{C\left[0, x_{0}\right]}<\frac{\epsilon}{3}, \quad \forall n \geq n_{1} .
$$

By using Lemma 2.1, we can find $n_{2} \in \mathbb{N}$ such that

$$
\begin{aligned}
& \left|L_{n}^{\alpha_{n}, \beta_{n}}\left(1+t^{2} ; x\right)-\left(1+x^{2}\right)\right|<\frac{\epsilon}{3\|f\|_{2}}, \quad \forall n \geq n_{2}, \quad \text { or, } \\
& L_{n}^{\alpha_{n}, \beta_{n}}\left(1+t^{2} ; x\right)<\left(1+x^{2}\right)+\frac{\epsilon}{3\|f\|_{2}}, \quad \forall n \geq n_{2} .
\end{aligned}
$$

Hence

$$
\begin{aligned}
I_{2} & =\|f\|_{2} \sup _{x>x_{0}} \frac{\left|L_{n}^{\alpha_{n}, \beta_{n}}\left(1+t^{2} ; x\right)\right|}{\left(1+x^{2}\right)^{1+r}} \\
& <\|f\|_{2} \sup _{x>x_{0}} \frac{1}{\left(1+x^{2}\right)^{1+r}}\left(\left(1+x^{2}\right)+\frac{\epsilon}{3\|f\|_{2}}\right), \quad \forall n \geq n_{2} \\
& <\|f\|_{2} \sup _{x>x_{0}}\left(\frac{1}{\left(1+x^{2}\right)^{r}}\right)+\frac{\epsilon}{3}, \quad \forall n \geq n_{2} \\
& <\frac{\|f\|_{2}}{\left(1+x_{0}^{2}\right)^{r}}+\frac{\epsilon}{3}, \quad \forall n \geq n_{2} .
\end{aligned}
$$

Now, using (13), we get

$$
I_{3}=\sup _{x>x_{0}} \frac{|f(x)|}{\left(1+x^{2}\right)^{1+r}} \leq \frac{\|f\|_{2}}{\left(1+x_{0}^{2}\right)^{r}} .
$$


Let $n_{0}=\max \left\{n_{1}, n_{2}\right\}$, then by (15), (16) and (17)

$$
I_{1}+I_{2}+I_{3}<\frac{2\|f\|_{2}}{\left(1+x_{0}^{2}\right)^{r}}+\frac{2 \epsilon}{3}, \quad \forall n \geq n_{0} .
$$

Choose $x_{0}$ to be so large that

$$
\frac{2\|f\|_{2}}{\left(1+x_{0}^{2}\right)^{r}}<\frac{\epsilon}{3}
$$

Then, combining (14), (18) and (19), we have

$$
\sup _{x \in[0, \infty)} \frac{\left|L_{n}^{\alpha_{n}, \beta_{n}}(f ; x)-f(x)\right|}{\left(1+x^{2}\right)^{1+r}}<\epsilon, \quad \forall n \geq n_{0} .
$$

Hence the proof is done.

Bojanic [23] studied the rate of convergence for Fourier series of functions of bounded variation. Cheng [24] obtained the rate of convergence of Bernstein polynomials of functions of bounded variation. Later on many researchers have made important contributions in this area of approximation theory (cf. [25-29] etc.). Bojanic and Cheng [30] studied the rate of convergence of Bernstein polynomials for functions with derivatives of bounded variation. Later on, Bojanic and Khan [31] estimated the rate of convergence of some operators of functions with derivatives of bounded variation. Shaw et al. [32] obtained the rates for approximation of functions of bounded variation and for functions with derivatives of bounded variation by positive linear operators. Many mathematicians studied in this direction and their work pertaining to this area is described in the papers [14, 26, 33-38] etc.

Now, we shall obtain the rate of convergence of $L_{n}^{\alpha_{n}, \beta_{n}}(f ; x)$ for functions having derivatives of bounded variation. Let $\operatorname{DBV}[0, \infty)$ be the space of functions in $C_{2}[0, \infty)$, which have the derivative of bounded variation on every finite subinterval of $[0, \infty)$. Here we show that at the points $x$, where $f^{\prime}(x+)$ and $f^{\prime}(x-)$ exist, the operator $L_{n}^{\alpha_{n}, \beta_{n}}(f ; x)$ converges to the function $f(x)$. A function $f \in \operatorname{DBV}[0, \infty)$ can be represented as

$$
f(x)=\int_{0}^{x} g(t) d t+f(0)
$$

where $g$ denotes a function of bounded variation on every finite subinterval of $[0, \infty)$.

In order to study the convergence of the operators $L_{n}^{\alpha_{n}, \beta_{n}}$ for the functions having a derivative of bounded variation, we rewrite the operator (1) as follows:

$$
L_{n}^{\alpha_{n}, \beta_{n}}(f ; x)=\int_{0}^{\infty} W(t, x) f(t) d t
$$

where $W(t, x)$ is the kernel given by

$$
W(t, x)=\frac{\beta_{n}}{A(1) B\left(\alpha_{n} x\right)} \sum_{k=0}^{\infty} p_{k}\left(\alpha_{n} x\right) \chi_{I}(t),
$$

$\chi_{I}(t)$ being the characteristic function of $I=\left[\frac{k}{\beta_{n}}, \frac{k+1}{\beta_{n}}\right]$. 
Lemma 4.2 Let for all $x>0$ and sufficiently large $n$

(1) $\lambda_{\alpha_{n}, \beta_{n}}(t, x)=\int_{0}^{t} W(u, x) d u \leq \frac{\delta_{\alpha_{n}, \beta_{n}}^{2}(x)}{(x-t)^{2}}, 0 \leq t<x$,

(2) $1-\lambda_{\alpha_{n}, \beta_{n}}(t, x)=\int_{t}^{\infty} W(u, x) d u \leq \frac{\delta_{\alpha_{n}, \beta_{n}}^{2}(x)}{(t-x)^{2}}, x \leq t<\infty$.

Proof Using Lemma 2.1 and the definition of kernel, we get

$$
\begin{aligned}
\lambda_{\alpha_{n}, \beta_{n}}(t, x) & =\int_{0}^{t} W(u, x) d u \\
& \leq \int_{0}^{t}\left(\frac{x-u}{x-t}\right)^{2} W(u, x) d u \\
& \leq \frac{1}{(x-t)^{2}} \int_{0}^{t}(u-x)^{2} W(u, x) d u .
\end{aligned}
$$

Hence

$$
\begin{aligned}
\lambda_{\alpha_{n}, \beta_{n}}(t, x) & \leq \frac{1}{(x-t)^{2}} L_{n}^{\alpha_{n}, \beta_{n}}\left((u-x)^{2} ; x\right) \\
& =\frac{1}{(x-t)^{2}} \delta_{\alpha_{n}, \beta_{n}}^{2}(x) .
\end{aligned}
$$

Similarly, we can prove the other inequality, therefore we omit the details.

Let $\bigvee_{a}^{b} f$ be the total variation of $f$ on $[a, b]$, i.e.,

$$
\bigvee_{a}^{b} f=V(f ;[a, b])=\sup _{P \in \mathbb{P}}\left(\sum_{i=1}^{n}\left|f\left(x_{i}\right)-f\left(x_{i-1}\right)\right|\right), \quad \text { bounded variation, }
$$

where $\mathbb{P}$ is the set of all partitions $P=\left\{a=x_{0}, x_{1}, \ldots, x_{n}=b\right\}$ of $[a, b]$ and it also has the property

$$
\bigvee_{a}^{b} f=\bigvee_{a}^{c} f+\bigvee_{c}^{b} f
$$

Let

$$
f_{x}^{\prime}(t)= \begin{cases}f^{\prime}(t)-f^{\prime}(x-), & 0 \leq t<x, \\ 0, & t=x, \\ f^{\prime}(t)-f^{\prime}(x+), & x<t<\infty\end{cases}
$$

Theorem 4.3 Let $f \in \operatorname{DBV}[0, \infty), x>0$ and $n$ be sufficiently large, then

$$
\begin{aligned}
\left|L_{n}^{\alpha_{n}, \beta_{n}}(f ; x)-f(x)\right| \leq & \left|\frac{1}{2}\left(f^{\prime}(x+)+f^{\prime}(x-)\right)\right|\left|\gamma_{\alpha_{n}, \beta_{n}}(x)\right| \\
& +\frac{\delta_{\alpha_{n}, \beta_{n}}^{2}(x)}{x} \sum_{k=1}^{[\sqrt{n}]}\left(\bigvee_{x-x / k}^{x+x / k} f_{x}^{\prime}\right)+\frac{x}{\sqrt{n}}\left(\bigvee_{x-x / \sqrt{n}}^{x+x / \sqrt{n}} f_{x}^{\prime}\right) \\
& +\frac{\delta_{\alpha_{n}, \beta_{n}}^{2}(x)}{x^{2}}\left|f(2 x)-f(x)-x f^{\prime}(x+)\right|
\end{aligned}
$$




$$
\begin{aligned}
& +\left(\frac{M_{f}+|f(x)|}{x^{2}}+4 M_{f}\right) \delta_{\alpha_{n}, \beta_{n}}^{2}(x)+\left|f^{\prime}(x+)\right| \delta_{\alpha_{n}, \beta_{n}}(x) \\
& +\left|\frac{1}{2}\left(f^{\prime}(x+)-f^{\prime}(x-)\right)\right| \delta_{\alpha_{n}, \beta_{n}}(x)
\end{aligned}
$$

Proof By hypothesis (22),

$$
\begin{aligned}
f^{\prime}(t)= & \frac{1}{2}\left(f^{\prime}(x+)+f^{\prime}(x-)\right)+f_{x}^{\prime}(t)+\frac{1}{2}\left(f^{\prime}(x+)-f^{\prime}(x-)\right) \operatorname{sgn}(t-x) \\
& +\delta_{x}(t)\left(f^{\prime}(t)-\frac{1}{2}\left(f^{\prime}(x+)+f^{\prime}(x-)\right)\right),
\end{aligned}
$$

where

$$
\delta_{x}(t)= \begin{cases}1, & t=x, \\ 0, & t \neq x\end{cases}
$$

Now, using Lemma 2.1, equations (20) and (23), we get

$$
\begin{aligned}
L_{n}^{\alpha_{n}, \beta_{n}}(f ; x)-f(x) \\
=\int_{0}^{\infty}(f(t)-f(x)) W(t, x) d t \\
=\int_{0}^{\infty}\left(\int_{x}^{t} f^{\prime}(u) d u\right) W(t, x) d t \\
=\int_{0}^{\infty}\left[\int _ { x } ^ { t } \left\{\frac{1}{2}\left(f^{\prime}(x+)+f^{\prime}(x-)\right)+f_{x}^{\prime}(u)+\frac{1}{2}\left(f^{\prime}(x+)-f^{\prime}(x-)\right) \operatorname{sgn}(u-x)\right.\right. \\
\left.\left.\quad+\delta_{x}(u)\left(f^{\prime}(u)-\frac{1}{2}\left(f^{\prime}(x+)+f^{\prime}(x-)\right)\right)\right\} d u\right] W(t, x) d t .
\end{aligned}
$$

Since $\int_{x}^{t} \delta_{x}(u) d u=0$

$$
\begin{aligned}
& L_{n}^{\alpha_{n}, \beta_{n}}(f ; x)-f(x) \\
& =\frac{1}{2}\left(f^{\prime}(x+)+f^{\prime}(x-)\right) \int_{0}^{\infty}(t-x) W(t, x) d t+\int_{0}^{\infty}\left(\int_{x}^{t} f_{x}^{\prime}(u) d u\right) W(t, x) d t \\
& \quad+\frac{1}{2}\left(f^{\prime}(x+)-f^{\prime}(x-)\right) \int_{0}^{\infty}|t-x| W(t, x) d t .
\end{aligned}
$$

Now, we break the second term on the right-hand side of the above equation as follows:

$$
\begin{aligned}
\int_{0}^{\infty}\left(\int_{x}^{t} f_{x}^{\prime}(u) d u\right) W(t, x) d t= & -\int_{0}^{x}\left(\int_{t}^{x} f_{x}^{\prime}(u) d u\right) W(t, x) d t \\
& +\int_{x}^{\infty}\left(\int_{x}^{t} f_{x}^{\prime}(u) d u\right) W(t, x) d t \\
= & -I_{1}+I_{2},
\end{aligned}
$$


where

$$
\begin{aligned}
& I_{1}=\int_{0}^{x}\left(\int_{t}^{x} f_{x}^{\prime}(u) d u\right) W(t, x) d t, \\
& I_{2}=\int_{x}^{\infty}\left(\int_{x}^{t} f_{x}^{\prime}(u) d u\right) W(t, x) d t .
\end{aligned}
$$

Taking the modulus on both sides of (24), we get

$$
\begin{aligned}
\left|L_{n}^{\alpha_{n}, \beta_{n}}(f ; x)-f(x)\right| \leq & \left|\frac{1}{2}\left(f^{\prime}(x+)+f^{\prime}(x-)\right)\right|\left|L_{n}^{\alpha_{n}, \beta_{n}}(t-x ; x)\right|+\left|I_{1}\right| \\
& +\left|I_{2}\right|+\left|\frac{1}{2}\left(f^{\prime}(x+)-f^{\prime}(x-)\right)\right| L_{n}^{\alpha_{n}, \beta_{n}}(|t-x| ; x) .
\end{aligned}
$$

After applying the Cauchy-Schwarz inequality, we get

$$
\begin{aligned}
\left|L_{n}^{\alpha_{n}, \beta_{n}}(f ; x)-f(x)\right| \leq & \left|\frac{1}{2}\left(f^{\prime}(x+)+f^{\prime}(x-)\right)\right|\left|\gamma_{\alpha_{n}, \beta_{n}}(x)\right|+\left|I_{1}\right|+\left|I_{2}\right| \\
& +\left|\frac{1}{2}\left(f^{\prime}(x+)-f^{\prime}(x-)\right)\right| \sqrt{L_{n}^{\alpha_{n}, \beta_{n}}\left((t-x)^{2} ; x\right)} \\
= & \left|\frac{1}{2}\left(f^{\prime}(x+)+f^{\prime}(x-)\right)\right|\left|\gamma_{\alpha_{n}, \beta_{n}}(x)\right|+\left|I_{1}\right|+\left|I_{2}\right| \\
& +\left|\frac{1}{2}\left(f^{\prime}(x+)-f^{\prime}(x-)\right)\right| \delta_{\alpha_{n}, \beta_{n}}(x) .
\end{aligned}
$$

Now applying Lemma 4.2 and integration by parts, $I_{1}$ can be written as

$$
\begin{aligned}
I_{1} & =\int_{0}^{x}\left(\int_{t}^{x} f_{x}^{\prime}(u) d u\right) W(t, x) d t \\
& =\int_{0}^{x}\left(\int_{t}^{x} f_{x}^{\prime}(u) d u\right) \frac{\partial}{\partial t} \lambda_{\alpha_{n}, \beta_{n}}(t, x) d t \\
& =\int_{0}^{x} f_{x}^{\prime}(t) \lambda_{\alpha_{n}, \beta_{n}}(t, x) d t .
\end{aligned}
$$

After taking the modulus, we have

$$
\begin{aligned}
\left|I_{1}\right| & \leq \int_{0}^{x}\left|f_{x}^{\prime}(t)\right| \lambda_{\alpha_{n}, \beta_{n}}(t, x) d t \\
& \leq \int_{0}^{x-x / \sqrt{n}}\left|f_{x}^{\prime}(t)\right| \lambda_{\alpha_{n}, \beta_{n}}(t, x) d t+\int_{x-x / \sqrt{n}}^{x}\left|f_{x}^{\prime}(t)\right| \lambda_{\alpha_{n}, \beta_{n}}(t, x) d t \\
& =K_{1}+K_{2}, \quad \text { say. }
\end{aligned}
$$

Since $f_{x}^{\prime}(x)=0$, by hypothesis $(22)$,

$$
K_{1}=\int_{0}^{x-x / \sqrt{n}}\left|f_{x}^{\prime}(t)-f_{x}^{\prime}(x)\right| \lambda_{\alpha_{n}, \beta_{n}}(t, x) d t
$$


Now, using Lemma 4.2, we get

$$
K_{1} \leq \delta_{\alpha_{n}, \beta_{n}}^{2}(x) \int_{0}^{x-x / \sqrt{n}}\left|f_{x}^{\prime}(t)-f_{x}^{\prime}(x)\right| \frac{d t}{(x-t)^{2}}
$$

By the definition of total variation (21) and taking $t=x-x / u$, we have

$$
\begin{aligned}
K_{1} & \leq \delta_{\alpha_{n}, \beta_{n}}^{2}(x) \int_{0}^{x-x / \sqrt{n}}\left(\bigvee_{t}^{x} f_{x}^{\prime}\right) \frac{d t}{(x-t)^{2}} \\
& =\delta_{\alpha_{n}, \beta_{n}}^{2}(x) \int_{1}^{\sqrt{n}}\left(\bigvee_{x-x / u}^{x} f_{x}^{\prime}\right) \frac{d u}{x} .
\end{aligned}
$$

Now, after breaking integral into summation,

$$
\begin{aligned}
K_{1} & \leq \frac{\delta_{\alpha_{n}, \beta_{n}}^{2}(x)}{x} \sum_{k=1}^{[\sqrt{n}]} \int_{k}^{k+1}\left(\bigvee_{x-x / u}^{x} f_{x}^{\prime}\right) d u \\
& \leq \frac{\delta_{\alpha_{n}, \beta_{n}}^{2}(x)}{x} \sum_{k=1}^{[\sqrt{n}]}\left(\bigvee_{x-x / k}^{x} f_{x}^{\prime}\right)\left(\int_{k}^{k+1} d u\right) \\
& =\frac{\delta_{\alpha_{n}, \beta_{n}}^{2}(x)}{x} \sum_{k=1}^{[\sqrt{n}]}\left(\bigvee_{x-x / k}^{x} f_{x}^{\prime}\right) .
\end{aligned}
$$

By Lemma $4.2, \lambda_{\alpha_{n}, \beta_{n}}(t, x) \leq 1$ and using hypothesis (22), we have

$$
\begin{aligned}
K_{2} & =\int_{x-x / \sqrt{n}}^{x}\left|f_{x}^{\prime}(t)\right| \lambda_{\alpha_{n}, \beta_{n}}(t, x) d t \\
& \leq \int_{x-x / \sqrt{n}}^{x}\left|f_{x}^{\prime}(t)-f_{x}^{\prime}(x)\right| d t .
\end{aligned}
$$

Now, by the definition of total variation (21),

$$
\begin{aligned}
K_{2} & \leq \int_{x-x / \sqrt{n}}^{x}\left(\bigvee_{t}^{x} f_{x}^{\prime}\right) d t \\
& \leq\left(\bigvee_{x-x / \sqrt{n}}^{x} f_{x}^{\prime}\right) \int_{x-x / \sqrt{n}}^{x} d t \\
& =\frac{x}{\sqrt{n}}\left(\bigvee_{x-x / \sqrt{n}}^{x} f_{x}^{\prime}\right) .
\end{aligned}
$$

Hence,

$$
\left|I_{1}\right| \leq \frac{\delta_{\alpha_{n}, \beta_{n}}^{2}(x)}{x} \sum_{k=1}^{[\sqrt{n}]}\left(\bigvee_{x-x / k}^{x} f_{x}^{\prime}\right)+\frac{x}{\sqrt{n}}\left(\bigvee_{x-x / \sqrt{n}}^{x} f_{x}^{\prime}\right)
$$


Gang et al. Journal of Inequalities and Applications (2017) 2017:156

Page 17 of 21

Using Lemma 4.2, we can write

$$
\begin{aligned}
\left|I_{2}\right|= & \left|\int_{x}^{\infty}\left(\int_{x}^{t} f_{x}^{\prime}(u) d u\right) W(t, x) d t\right| \\
\leq & \left|\int_{x}^{2 x}\left(\int_{x}^{t} f_{x}^{\prime}(u) d u\right) \frac{\partial}{\partial t}\left(1-\lambda_{\alpha_{n}, \beta_{n}}(t, x)\right) d t\right| \\
& +\left|\int_{2 x}^{\infty}\left(\int_{x}^{t} f_{x}^{\prime}(u) d u\right) W(t, x) d t\right| .
\end{aligned}
$$

Now, applying integration by parts and hypothesis (22), we obtain

$$
\begin{aligned}
\left|I_{2}\right| \leq & \left|\int_{x}^{2 x} f_{x}^{\prime}(u) d u \cdot\left(1-\lambda_{\alpha_{n}, \beta_{n}}(2 x, x)\right)-\int_{x}^{2 x} f_{x}^{\prime}(t)\left(1-\lambda_{\alpha_{n}, \beta_{n}}(t, x)\right) d t\right| \\
& +\left|\int_{2 x}^{\infty}\left(\int_{x}^{t}\left(f^{\prime}(u)-f^{\prime}(x+)\right) d u\right) W(t, x) d t\right| \\
\leq & \left|\int_{x}^{2 x} f_{x}^{\prime}(u) d u\right| \cdot \frac{\delta_{\alpha_{n}, \beta_{n}}^{2}(x)}{(x)^{2}}+\int_{x}^{2 x}\left|f_{x}^{\prime}(t)\right| \cdot\left(1-\lambda_{\alpha_{n}, \beta_{n}}(t, x)\right) d t \\
& +\left|\int_{2 x}^{\infty}(f(t)-f(x)) W(t, x) d t\right|+\left|f^{\prime}(x+)\right|\left|\int_{2 x}^{\infty}(t-x) W(t, x) d t\right| \\
= & P_{1}+P_{2}+P_{3}+P_{4}, \quad \text { say. }
\end{aligned}
$$

Now, by hypothesis (22),

$$
\begin{aligned}
P_{1} & =\frac{\delta_{\alpha_{n}, \beta_{n}}^{2}(x)}{x^{2}}\left|\int_{x}^{2 x} f_{x}^{\prime}(u) d u\right| \\
& \leq \frac{\delta_{\alpha_{n}, \beta_{n}}^{2}(x)}{x^{2}}\left|\int_{x}^{2 x}\left(f^{\prime}(u)-f^{\prime}(x+)\right) d u\right| \\
& \leq \frac{\delta_{\alpha_{n}, \beta_{n}}^{2}(x)}{x^{2}}\left|f(2 x)-f(x)-x f^{\prime}(x+)\right|
\end{aligned}
$$

and

$$
\begin{aligned}
P_{2}= & \int_{x}^{2 x}\left|f_{x}^{\prime}(t)\right| \cdot\left(1-\lambda_{\alpha_{n}, \beta_{n}}(t, x)\right) d t \\
= & \int_{x}^{x+x / \sqrt{n}}\left|f_{x}^{\prime}(t)\right| \cdot\left(1-\lambda_{\alpha_{n}, \beta_{n}}(t, x)\right) d t \\
& +\int_{x+x \sqrt{n}}^{2 x}\left|f_{x}^{\prime}(t)\right| \cdot\left(1-\lambda_{\alpha_{n}, \beta_{n}}(t, x)\right) d t \\
= & J_{1}+J_{2}, \quad \text { say. }
\end{aligned}
$$

Using Lemma $4.2,1-\lambda_{\alpha_{n}, \beta_{n}}(t, x) \leq 1$ and hypothesis (22), we get

$$
\begin{aligned}
J_{1} & =\int_{x}^{x+x / \sqrt{n}}\left|f_{x}^{\prime}(t)\right| \cdot\left(1-\lambda_{\alpha_{n}, \beta_{n}}(t, x)\right) d t \\
& \leq \int_{x}^{x+x / \sqrt{n}}\left|f_{x}^{\prime}(t)-f_{x}^{\prime}(x)\right| d t
\end{aligned}
$$




$$
\begin{aligned}
& \leq \int_{x}^{x+x / \sqrt{n}}\left(\bigvee_{x}^{t} f_{x}^{\prime}\right) d t \\
& \leq\left(\bigvee_{x}^{x+x / \sqrt{n}} f_{x}^{\prime}\right) \int_{x}^{x+x / \sqrt{n}} d t \\
& \leq \frac{x}{\sqrt{n}}\left(\bigvee_{x}^{x+x / \sqrt{n}} f_{x}^{\prime}\right)
\end{aligned}
$$

Now, again with the help of Lemma 4.2 and by hypothesis (22), we get

$$
\begin{aligned}
J_{2} & =\int_{x+x \sqrt{n}}^{2 x}\left|f_{x}^{\prime}(t)\right| \cdot\left(1-\lambda_{\alpha_{n}, \beta_{n}}(t, x)\right) d t \\
& \leq \delta_{\alpha_{n}, \beta_{n}}^{2}(x) \int_{x+x \sqrt{n}}^{2 x}\left|f_{x}^{\prime}(t)-f_{x}^{\prime}(x)\right| \frac{d t}{(t-x)^{2}} .
\end{aligned}
$$

By using (21) and $t=x+x / u$,

$$
\begin{aligned}
J_{2} & \leq \delta_{\alpha_{n}, \beta_{n}}^{2}(x) \int_{x+x \sqrt{n}}^{2 x}\left(\bigvee_{x}^{t} f_{x}^{\prime}\right) \frac{d t}{(t-x)^{2}} \\
& =\delta_{\alpha_{n}, \beta_{n}}^{2}(x) \int_{1}^{\sqrt{n}}\left(\bigvee_{x}^{x+x / u} f_{x}^{\prime}\right) \frac{d u}{x} \\
& \leq \frac{\delta_{\alpha_{n}, \beta_{n}}^{2}(x)}{x} \sum_{k=1}^{[\sqrt{n}]} \int_{k}^{k+1}\left(\bigvee_{x}^{x+x / u} f_{x}^{\prime}\right) d u \\
& \leq \frac{\delta_{\alpha_{n}, \beta_{n}}^{2}(x)}{x} \sum_{k=1}^{[\sqrt{n}]}\left(\bigvee_{x}^{x+x / k} f_{x}^{\prime}\right)\left(\int_{k}^{k+1} d u\right) \\
& =\frac{\delta_{\alpha_{n}, \beta_{n}}^{2}(x)}{x} \sum_{k=1}^{[\sqrt{n}]}\left(\bigvee_{x}^{x+x / k} f_{x}^{\prime}\right) .
\end{aligned}
$$

Hence,

$$
P_{2} \leq \frac{x}{\sqrt{n}}\left(\bigvee_{x}^{x+x / \sqrt{n}} f_{x}^{\prime}\right)+\frac{\delta_{\alpha_{n}, \beta_{n}}^{2}(x)}{x} \sum_{k=1}^{[\sqrt{n}]}\left(\bigvee_{x}^{x+x / k} f_{x}^{\prime}\right)
$$

Now, using the Cauchy-Schwarz inequality, we estimate $P_{4}$

$$
\begin{aligned}
P_{4} & =\left|f^{\prime}(x+)\right|\left|\int_{2 x}^{\infty}(t-x) W(t, x) d t\right| \\
& \leq\left|f^{\prime}(x+)\right| \int_{2 x}^{\infty}|t-x| W(t, x) d t \\
& \leq\left|f^{\prime}(x+)\right| \int_{0}^{\infty}|t-x| W(t, x) d t \\
& =\left|f^{\prime}(x+)\right| \sqrt{L_{n}^{\alpha_{n}, \beta_{n}}\left((t-x)^{2} ; x\right)} \\
& =\left|f^{\prime}(x+)\right| \delta_{\alpha_{n}, \beta_{n}}(x) .
\end{aligned}
$$


Further, as $t \geq 2 x$, then $2(t-x) \geq t$ and $t-x \geq x$ gives

$$
\begin{aligned}
P_{3}= & \left|\int_{2 x}^{\infty}(f(t)-f(x)) W(t, x) d t\right| \\
\leq & \int_{2 x}^{\infty}|f(t)| W(t, x) d t+\int_{2 x}^{\infty}|f(x)| W(t, x) d t \\
\leq & M_{f} \int_{2 x}^{\infty}\left(1+t^{2}\right) W(t, x) d t+|f(x)| \int_{2 x}^{\infty} W(t, x) d t \\
= & \left(M_{f}+|f(x)|\right) \int_{2 x}^{\infty} W(t, x) d t+M_{f} \int_{2 x}^{\infty} t^{2} W(t, x) d t \\
\leq & \left(M_{f}+|f(x)|\right) \int_{2 x}^{\infty} \frac{(t-x)^{2}}{x^{2}} W(t, x) d t \\
& +M_{f} \int_{2 x}^{\infty} 4(t-x)^{2} W(t, x) d t \\
\leq & \left(\frac{M_{f}+|f(x)|}{x^{2}}+4 M_{f}\right) \int_{2 x}^{\infty}(t-x)^{2} W(t, x) d t \\
\leq & \left(\frac{M_{f}+|f(x)|}{x^{2}}+4 M_{f}\right) L_{n}^{\alpha_{n}, \beta_{n}}\left((t-x)^{2} ; x\right) \\
= & \left(\frac{M_{f}+|f(x)|}{x^{2}}+4 M_{f}\right) \delta_{\alpha_{n}, \beta_{n}}^{2}(x) .
\end{aligned}
$$

Hence,

$$
\begin{aligned}
\left|I_{2}\right| \leq & \frac{\delta_{\alpha_{n}, \beta_{n}}^{2}(x)}{x^{2}}\left|f(2 x)-f(x)-x f^{\prime}(x+)\right| \\
& +\frac{x}{\sqrt{n}}\left(\bigvee_{x}^{x+x / \sqrt{n}} f_{x}^{\prime}\right)+\frac{\delta_{\alpha_{n}, \beta_{n}}^{2}(x)}{x} \sum_{k=1}^{[\sqrt{n}]}\left(\bigvee_{x}^{x+x / k} f_{x}^{\prime}\right) \\
& +\left(\frac{M_{f}+|f(x)|}{x^{2}}+4 M_{f}\right) \delta_{\alpha_{n}, \beta_{n}}^{2}(x)+\left|f^{\prime}(x+)\right| \delta_{\alpha_{n}, \beta_{n}}(x) .
\end{aligned}
$$

Now from (25)-(27) we obtain

$$
\begin{aligned}
\left|L_{n}^{\alpha_{n}, \beta_{n}}(f ; x)-f(x)\right| \leq & \frac{1}{2}\left(f^{\prime}(x+)+f^{\prime}(x-)\right)|| \gamma_{\alpha_{n}, \beta_{n}}(x)|+| I_{1}|+| I_{2} \mid \\
& +\left|\frac{1}{2}\left(f^{\prime}(x+)-f^{\prime}(x-)\right)\right| \delta_{\alpha_{n}, \beta_{n}}(x) \\
\leq & \frac{1}{2}\left(f^{\prime}(x+)+f^{\prime}(x-)\right)|| \gamma_{\alpha_{n}, \beta_{n}}(x) \mid \\
& +\frac{\delta_{\alpha_{n}, \beta_{n}}^{2}(x)}{x} \sum_{k=1}^{[\sqrt{n}]}\left(\bigvee_{x-x / k}^{x} f_{x}^{\prime}\right)+\frac{x}{\sqrt{n}}\left(\bigvee_{x-x / \sqrt{n}}^{x} f_{x}^{\prime}\right) \\
& +\frac{\delta_{\alpha_{n}, \beta_{n}}^{2}(x)}{x^{2}}\left|f(2 x)-f(x)-x f^{\prime}(x+)\right| \\
& +\frac{x}{\sqrt{n}}\left(\bigvee_{x}^{x+x / \sqrt{n}} f_{x}^{\prime}\right)+\frac{\delta_{\alpha_{n}, \beta_{n}}^{2}(x)}{x} \sum_{k=1}^{[\sqrt{n}]}\left(\bigvee_{x}^{x+x / k} f_{x}^{\prime}\right)
\end{aligned}
$$




$$
\begin{aligned}
& +\left(\frac{M_{f}+|f(x)|}{x^{2}}+4 M_{f}\right) \delta_{\alpha_{n}, \beta_{n}}^{2}(x)+\left|f^{\prime}(x+)\right| \delta_{\alpha_{n}, \beta_{n}}(x) \\
& +\left|\frac{1}{2}\left(f^{\prime}(x+)-f^{\prime}(x-)\right)\right| \delta_{\alpha_{n}, \beta_{n}}(x),
\end{aligned}
$$

which finally gives the required result.

\section{Conclusion}

We study the order of approximation of the Kantorovich-Szász type operators based on Brenke type polynomials with the aid of Peetre's K-functional and the Ditzian-Totik modulus of smoothness. The rate of convergence of these operators for functions in a Lipschitz type space and a weighted space is investigated. The degree of approximation of functions whose derivatives coincide a.e. with a function of bounded variation is also discussed.

\section{Acknowledgements}

The authors are extremely grateful to the reviewers for their critical reading of the manuscript of our paper and making valuable comments leading to a better presentation of the paper. The first author is thankful to the Ministry of Human Resources and Development, India for the financial support to carry out the above work, and the third author of this paper is supported by the Research Fund of Hasan Kalyoncu University, Turkey in 2017.

\section{Competing interests}

The authors declare that they have no competing interests.

\section{Authors' contributions}

All authors have equally contributed to this work.

\section{Author details}

'Department of Mathematics, Indian Institute of Technology Roorkee, Roorkee, 247667, India. ${ }^{2}$ Department of Economics, Faculty of Economics, Administrative and Social Sciences, Hasan Kalyoncu University, Gaziantep, 27410, Turkey.

\section{Publisher's Note}

Springer Nature remains neutral with regard to jurisdictional claims in published maps and institutional affiliations.

Received: 24 April 2017 Accepted: 16 June 2017 Published online: 29 June 2017

\section{References}

1. Szász, O: Generalization of S. Bernstein's polynomials to the infinite interval. J. Res. Natl. Bur. Stand. 45, $239-245$ (1950)

2. Jakimovski, A, Leviatan, D: Generalized Szász operators for the approximation in the infinite interval. Mathematica 11(34), 97-103 (1969)

3. Ismail, M: On a generalization of Szász operators. Mathematica 16(39)(2), 259-267 (1974)

4. Varma, S, Sucu, S, İçöz, G: Generalization of Szász operators involving Brenke type polynomials. Comput. Math. Appl. 64(2), 121-127 (2012)

5. Varma, S, Taşdelen, F: Szász type operators involving Charlier polynomials. Math. Comput. Model. 56(5-6), 118-122 (2012)

6. Taşdelen, F, Aktaş, R, Altin, A: A Kantorovich type of Szász operators including Brenke-type polynomials. Abstr. Appl. Anal. 2012, Article ID 867203 (2012)

7. Öksüzer, Ö, Karsli, H, Taşdelen, F: Approximation by a Kantorovich variant of Szász operators based on Brenke-type polynomials. Mediterr. J. Math. 13(5), 3327-3340 (2016)

8. Aktaş, R, Çekim, B, Taşdelen, F: A Kantorovich-Stancu type generalization of Szász operators including Brenke type polynomials. J. Funct. Spaces Appl. 2013, Article ID 935430 (2013)

9. Mursaleen, M, Ansari, KJ: On Chlodowsky variant of Szász operators by Brenke type polynomials. Appl. Math. Comput. 271, 991-1003 (2015)

10. Atakut, Ç, Büyükyazici, I: Approximation by Kantorovich-Szász type operators based on Brenke type polynomials. Numer. Funct. Anal. Optim. 37(12), 1488-1502 (2016)

11. İspir, N, Atakut, Ç: Approximation by modified Szász-Mirakjan operators on weighted spaces. Proc. Indian Acad. Sci. Math. Sci. 112(4), 571-578 (2002)

12. Kajla, A, Agrawal, PN: Approximation properties of Szász type operators based on Charlier polynomials. Turk. J. Math. 39(6), 990-1003 (2015)

13. Sucu, S, Büyükyazici, İ: Integral operators containing Sheffer polynomials. Bull. Math. Anal. Appl. 4(4), 56-66 (2012)

14. Kajla, A, Acu, AM, Agrawal, PN: Baskakov-Szász-type operators based on inverse Pólya-Eggenberger distribution. Ann. Funct. Anal. 8(1), 106-123 (2017)

15. Neer, T, Agrawal, PN, Araci, S: Stancu-Durrmeyer type operators based on q-integers. Appl. Math. Inf. Sci. 11(3), 767-775 (2017) 
16. Özarslan, MA, Duman, O: Local approximation behavior of modified SMK operators. Miskolc Math. Notes 11(1), 87-99 (2010)

17. Lenze, B: On Lipschitz-type maximal functions and their smoothness spaces. Ned. Akad. Wet., Indag. Math. 50(1), 53-63 (1988)

18. Kajla, A: Direct estimates of certain Miheşan-Durrmeyer type operators. Adv. Oper. Theory 2(2), 162-178 (2017)

19. Ditzian, Z, Totik, V: Moduli of Smoothness. Springer Series in Computational Mathematics, vol. 9. Springer, New York (1987)

20. Aral, A, Acar, T: Weighted approximation by new Bernstein-Chlodowsky-Gadjiev operators. Filomat 27(2), $371-380$ (2013)

21. İspir, N: On modified Baskakov operators on weighted spaces. Turk. J. Math. 25(3), 355-365 (2001)

22. Yuksel, I, İspir, N: Weighted approximation by a certain family of summation integral-type operators. Comput. Math Appl. 52(10-11), 1463-1470 (2006)

23. Bojanic, R: An estimate of the rate of convergence for Fourier series of functions of bounded variation. Publ. Inst. Math. (Belgr.) 26(40), 57-60 (1979)

24. Cheng, F: On the rate of convergence of Bernstein polynomials of functions of bounded variation. J. Approx. Theory 39(3), 259-274 (1983)

25. Guo, SS, Khan, MK: On the rate of convergence of some operators on functions of bounded variation. J. Approx. Theory 58, 90-101 (1989)

26. Gupta, MK, Beniwal, MS, Goel, P: Rate of convergence for Szász-Mirakyan-Durrmeyer operators with derivatives of bounded variation. Appl. Math. Comput. 199(2), 828-832 (2008)

27. Öksüzer, Ö, Karsli, H, Taşdelen, F: Convergence rate of Bézier variant of an operator involving Laguerre polynomials of degree n. AIP Conf. Proc. 1558, 1160-1163 (2013)

28. Öksüzer, Ö, Karsli, H, Taşdelen, F: Order of approximation by an operator involving biorthogonal polynomials. J. Inequal. Appl. 2015, 121 (2015)

29. Öksüzer, Ö, Karsli, H, Taşdelen, F: Rate of convergence of the Bézier variant of an operator involving Laguerre polynomials. Math. Methods Appl. Sci. (2016). doi:10.1002/mma.3705

30. Bojanic, R, Cheng, F: Rate of convergence Bernstein polynomials for functions with derivatives of bounded variation. J. Math. Anal. Appl. 141(1), 136-151 (1989)

31. Bojanic, R, Khan, MK: Rate of convergence of some operators of functions with derivatives of bounded variation. Atti Semin. Mat. Fis. Univ. Modena 39(2), 495-512 (1991)

32. Shaw, SY, Liaw, WC, Lin, YL: Rates for approximation of functions in BV $[a, b]$ and DBV $[a, b]$ by positive linear operators. Chin. J. Math. 21, 171-193 (1993)

33. Acar, T, Gupta, V, Aral, A: Rate of convergence for generalized Szász operators. Bull. Math. Sci. 1(1)، 99-113 (2011)

34. Özarslan, MA, Duman, O, Kaanoğlu, C: Rates of convergence of certain King-type operators for functions with derivative of bounded variation. Math. Comput. Model. 52(1-2), 334-345 (2010)

35. Dhamija, M, Deo, N: Jain-Durrmeyer operators associated with the inverse Pólya-Eggenberger distribution. Appl. Math. Comput. 286, 15-22 (2016)

36. İspir, N: Rate of convergence of generalized rational type Baskakov operators. Math. Comput. Model. 46(5-6), 625-631 (2007)

37. Karsli, $\mathrm{H}$ : Rate of convergence of new gamma type operators for functions with derivatives of bounded variation. Math. Comput. Model. 45(5-6), 617-624 (2007)

38. Neer, T, Acu, AM, Agrawal, PN: Bézier variant of genuine-Durrmeyer type operators based on Pólya distribution. Carpath. J. Math. 33(1), 73-86 (2017)

\section{Submit your manuscript to a SpringerOpen ${ }^{\circ}$ journal and benefit from:}

- Convenient online submission

- Rigorous peer review

- Open access: articles freely available online

- High visibility within the field

- Retaining the copyright to your article

Submit your next manuscript at $>$ springeropen.com 